\title{
Aging Effects in the Central Jet Chamber of the H1 Experiment
}

Carsten Niebuhr

DESY, Notkestr. 85, 22607 Hamburg, Germany

\section{The H1 Tracking System}

The H1 detector [1] is a nearly hermetic multipurpose apparatus built to investigate the inelastic high-energy interactions of electrons and protons at the HERA ep collider. Closest to the interaction point are the central and forward tracking systems (see figure 1), which are surrounded

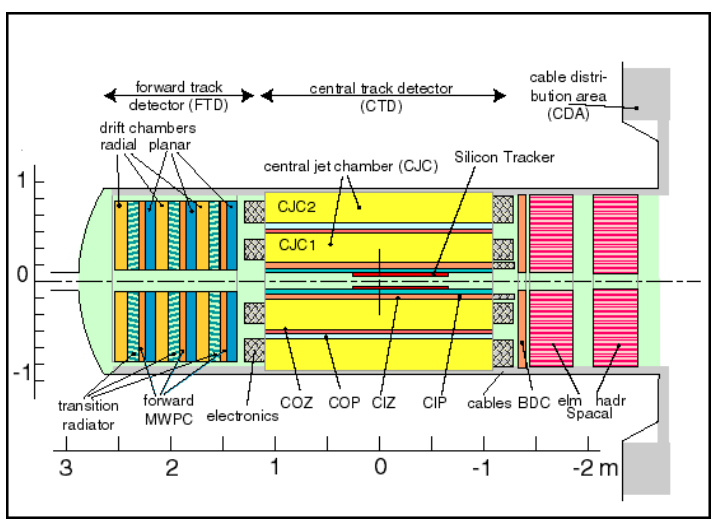

Figure 1. Cross section of the H1 track detectors.

by a liquid argon calorimeter consisting of an electromagnetic and a hadronic section. A superconducting solenoid surrounding both the tracking system and the calorimeter provides a uniform magnetic field of $1.15 \mathrm{~T}$ parallel to the beam line. The central jet chamber (CJC) consists of two concentric rings covering polar angles of $15^{\circ}$ to $165^{\circ}$. It is supplemented by two cylindrical drift chambers at radii of 18 and $47 \mathrm{~cm}$ to determine the $z$-coordinate of the tracks along the wire direction. A cylindrical proportional chamber is attached to each of those $z$-drift chambers for triggering.

\subsection{Parameters and Operation of the CJC}

Details of the construction and the readout of the CJC can be found elsewhere [1,2], here only those characteristics are summarized which are relevant for this study.

The inner ring of the CJC, CJC 1, has 24 layers of sense wires strung parallel to the beam axis and covers the radial range $20<r<45 \mathrm{~cm}$, while the outer ring, CJC 2, consists of 32 layers covering the range $53<r<85 \mathrm{~cm}$. For most of the running period from $1992-1998$ an Argon(50\%)/Ethane(50\%) gas mixture with $0.1 \%$ $\mathrm{H}_{2} \mathrm{O}$ as additive was used (only until May 1999, see section 4 for modifications).

CJC 1 and CJC 2 are connected to one common gas circuit[3]. Gas is circulated through the chambers in parallel at a flow corresponding to 1 - 2 chamber volume exchanges per day. Fresh gas is mixed into the system at a rate of $500 \mathrm{l}$ /day. The closed loop circuit is supplied with purification units for removing oxygen, (water) and low concentrations of higher organic compounds, which are removed at room temperature by a combination of $1.5 \mathrm{~kg} 3$ Angstroem and $0.5 \mathrm{~kg}$ 5 Angstroem molecular sieves. Hygrometers and an $\mathrm{O}_{2}$-trace analyzer allow an early detection of leaks in addition to the complete analysis of the gas composition which is performed by an automatic gas chromatograph station about twice per day. The sensitivity of this device is about 300 ppm.

The chamber is operated at a gas gain of $1-2$ $\cdot 10^{4}$. Under normal operating conditions and low background the chamber current in both chambers scales almost linearly with the current in the HERA-e ring (positrons or electrons). Typical values in 1997 for a HERA positron current of $30 \mathrm{~mA}$ were about $25 \mu \mathrm{A}$ in each of the chambers (see section 4 for deviations from these values for 
Table 1

Parameters of CJC 1 and CJC 2

\begin{tabular}{lcc}
\hline & CJC 1 & CJC 2 \\
\hline nr. of cells & 30 & 60 \\
\hline nr. of wires per cell & & 32 \\
$\ldots$. sense wires & 24 & 66 \\
$\ldots$ potential wires & 50 & 75 \\
$\ldots$. cathode/field wires & 59 & \\
chamber dimensions & & 544.8 \\
inner radius / mm & 218.3 & 823.2 \\
outer radius / mm & 425.8 & 2200 \\
active length / mm & 2200 & 3.08 \\
gas volume / $\mathrm{m}^{3}$ & 1.14 &
\end{tabular}

wire material and diameter

... sense wires

$20 \mu \mathrm{m}$ gold-plated $\mathrm{W}(+2 \% \mathrm{Re})$ (first and last sense wire: $25 \mu \mathrm{m}$ )

... potential wires

$127 \mu \mathrm{m} \mathrm{Cu}(+2 \% \mathrm{Be})$

... cathode/field wires

$180 \mu \mathrm{m} \mathrm{Cu}(+2 \% \mathrm{Be})$

gas system closed common circuit with purifier (molesieve 5A / 3A + Deoxo)

gas flow exchange rate $\approx 1-2$ volumes / day

gas tubing

$\approx 150 \mathrm{~m} \mathrm{Cu}$ and stainless steel gas tubing

gas mixture

standard ep running $\quad 50 \% \mathrm{Ar}+50 \% \mathrm{C}_{2} \mathrm{H}_{6}+0.1 \% \mathrm{H}_{2} \mathrm{O}\left(\geq\right.$ May 1999: $\left.\rightarrow 0.8 \% \mathrm{C}_{2} \mathrm{H}_{5} \mathrm{OH}\right)$

in other periods

$89.5 \% \mathrm{Ar}+9.5 \% \mathrm{CO}_{2}+1 \% \mathrm{CH}_{4}$

electron running).

\section{Wire Corrosion}

Already after the first few months of operation at HERA several sense wires of CJC 1 spontaneously broke during a maintenance operation in the shutdown 1992/1993. The overall HERA schedule did not allow opening of the chamber. Therefore, the broken wires only could be pulled out of CJC 1 to minimize the regions which were affected by shorts and the chamber was put back into operation again. Subsequently, more wires broke so that over a period of 7 months in total 48 wires broke, randomly distributed in CJC 1, while no damage was observed in CJC 2. The wires were found broken at the chamber end walls close to the point where they are fixed with crimp parts. At the tip of the crimp part a little brass insert with a $60 \mu \mathrm{m}$ diameter bore acts as wire feedthrough. Detailed investigations of both the broken wires and the crimp parts were performed using raster electron microscopy with x-ray micro analysis (REM/RMA) and laser mass spectroscopy (LAMMA [4]), the latter giving sensitivity to elements down to hydrogen.

Figure 2a shows that there are lots of impurities around the $60 \mu \mathrm{m}$ bore of the brass insert with sharp edges causing damage of the gold layer on the wire surface. The impurities are probably remnants from the gilding process of the crimp parts. The tungsten wire is therefore exposed to complex chemical reactions as can be inferred from the LAMMA spectrum in figure $2 \mathrm{c}$ of one of the wires which shows the presence of a large variety of chemical compounds involving other metals such as Na, Ca, and K. Particularly important are characteristic peaks between 300 and 400 atomic 

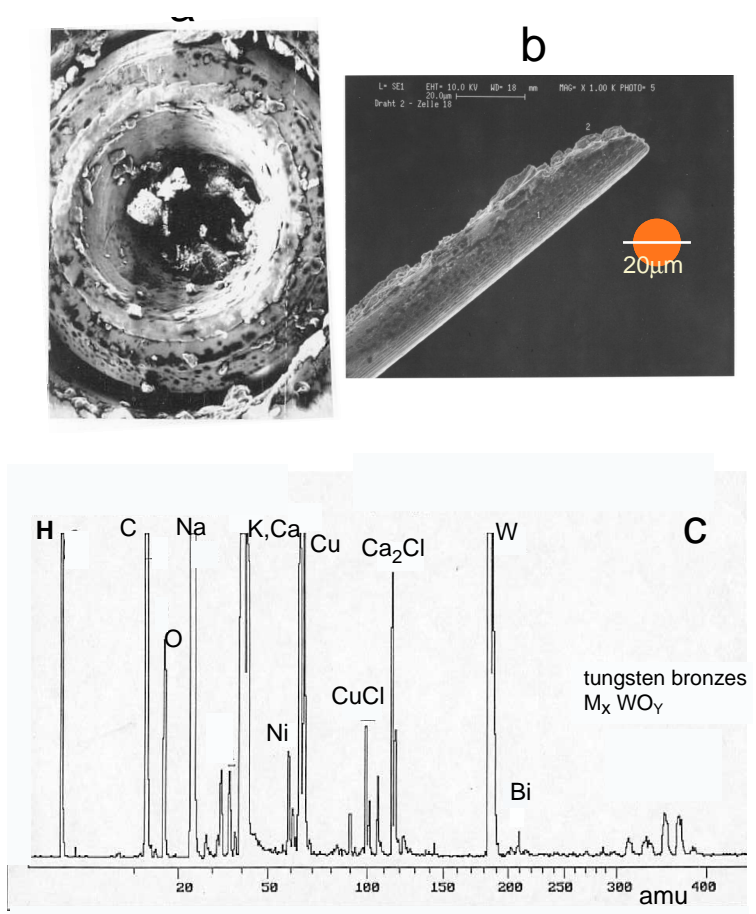

Figure 2. (a) $60 \mu \mathrm{m}$ bore of wire feedthrough with remnants from gilding process. (b) end of a broken wire. (c) LAMMA spectrum of wire end indicates the presence of hydrogen and tungsten bronzes.

mass units (amu) which suggest the formation of tungsten bronzes (of the form $\mathrm{M}_{X} \mathrm{WO}_{Y}$ where $\mathrm{M}$ stands for the metal) and a peak at $1 \mathrm{amu}$ which indicates the occurrence of hydrogen. Tungsten bronzes are known to become brittle when they dissolve sufficient amount of hydrogen. Taking also into account the observed fracture morphology (figure $2 \mathrm{~b}$ ) the most likely explanation for the effect is hydrogen-induced brittle fracture initiated by chemical reactions with remnants from the gilding process. However, it remains an open question why no such damage was observed in CJC 2 although the manufacturing process for both chambers was identical.

As a consequence, the whole production and cleaning process as well as the quality control of the crimp parts were significantly improved. In

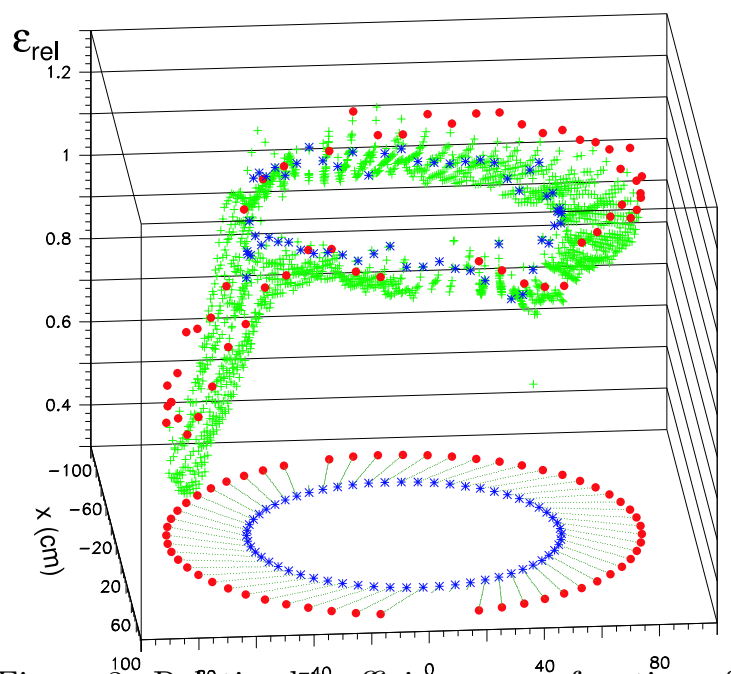

Figure 3. Reflative hit efficiency as a function of wire position. The first and last wires in each cell (25 $\mu \mathrm{m}$ diameter) operate at lower gas gain and are shown with different symbols.

the new design the brass inserts were replaced by jewels as are commonly used in watches. In the shutdown 1993/1994 all sense wires of CJC 1 were replaced and no further corrosive wire damage has been observed since then.

\section{Sense Wire Deposits}

In 1996 a small $\varphi$-dependence of the gas gain was observed in CJC 2 and to a smaller extent also in CJC 1. This was interpreted as a result of the increased top-bottom temperature gradient inside the tracking volume following an upgrade of the backward region of the H1 detector. Temperatures ranging from 30 to 45 degrees Celsius were measured with sensors placed at the inner face of the CJC end walls. A compensation of the gain differences was made by local $\mathrm{HV}$ adjustments in summer 1996. While the situation in CJC 1 was stable, the top-bottom asymmetry in CJC 2 continuously worsened during 1996/1997 although the corresponding integrated charge on wires of CJC 2 was lower than on wires of CJC 1 and was estimated to be $<0.01 \mathrm{C} / \mathrm{cm}$. Due to the larger radial distance to the beampipe and the larger number of cells the current per wire is 


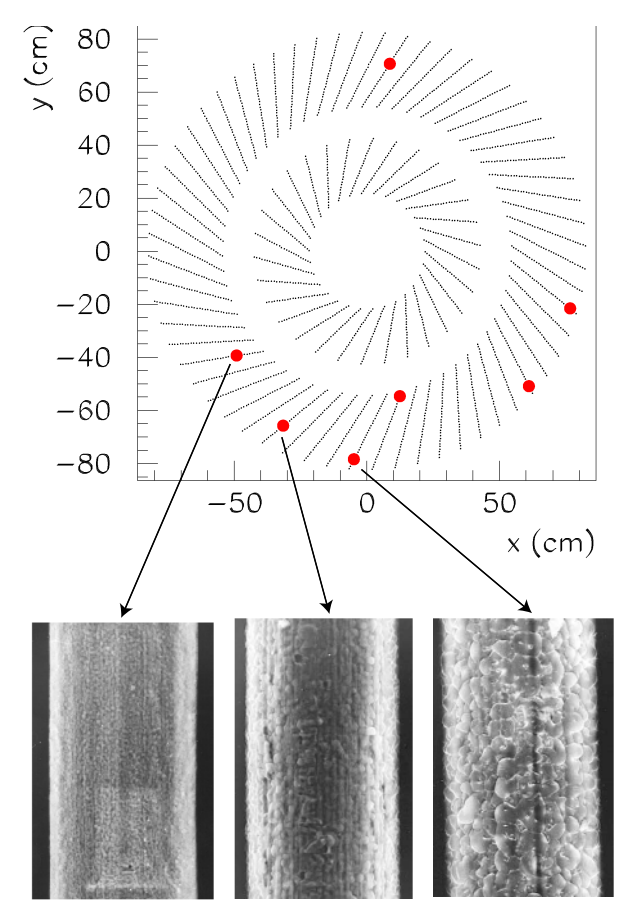

Figure 4. Wires from three different heights within the affected region of CJC 2. The amount of deposits depends on the height of the wire in the chamber.

typically a factor of 3 smaller in CJC 2 than in CJC 1.

The situation at the end of the 1997 running period is graphically represented in figure 3, which shows the relative hit efficiency for CJC 2 wires as a function of their position in the chamber. A strong correlation between efficiency and $y$-coordinate (height) of the affected wire is observed. While for wires above $y=-30 \mathrm{~cm}$ there is no sign of degradation visible, the wires below this level show an almost linear dependence of efficiency on height reaching relative hit efficiencies of about $30 \%$ at the lowest point in the chamber. Wires were removed from the chamber and scanned using an electron microscope[5]. As an example, pictures of three wires together with a sketch indicating their original position in CJC 2 are shown in figure 4 . The wires are coated
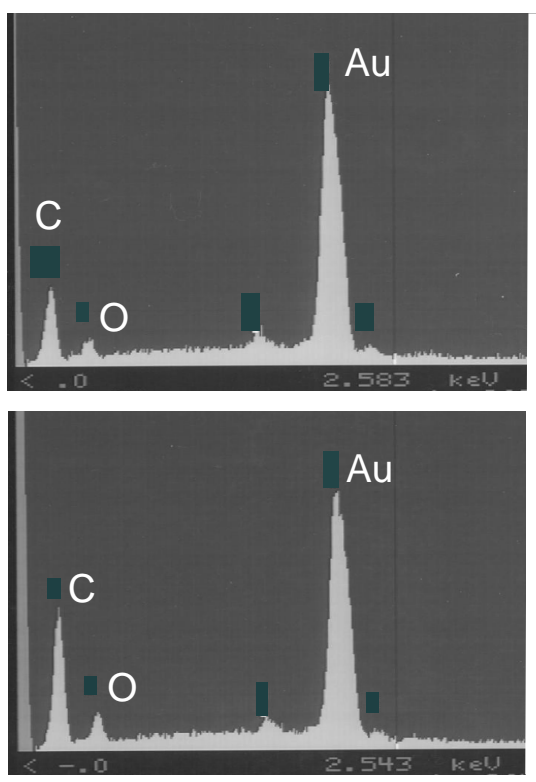

Figure 5. Energy-dispersive analysis of X-ray spectra (EDAX) taken from two different wires.

with deposits, which get more prominent for wires closer to the bottom of the chamber. In order to quantify the effect, an energy-dispersive analysis of x-ray spectra was performed. Figure 5 shows the spectra of two different wires. Normally, just a prominent gold peak is observed while wires from the affected region of CJC 2 show additional clear peaks of carbon and oxygen. No evidence of silicon is found on the sense wires, nor are any deposits seen on the adjacent potential and cathode wires. The abundance of carbon and oxygen relative to gold was estimated by forming the peak ratios for a rough quantitative measure. A monotonous relationship between y-coordinate of the wire, hit efficiency and relative abundance of carbon and oxygen is observed and is displayed in figure 6. Some amount of carbon and oxygen is already detected on a wire close to the knee in the efficiency plot which does not yet show a degradation of hit efficiency.

Although aging was only observed in the lower part of CJC 2, all sense wires of CJC 2 were replaced in the shutdown 1997/1998 making use of the improved design of the crimp parts devel- 
oped for the repair of CJC 1 in 1993 (see section 2). The striking dependence of the amount of aging on the height of the wire in the chamber strongly suggests an influence of impurities in the gas with a gradient in concentration due to gravitational and/or temperature effects possibly in combination with an insufficient gas flow through the chamber. Until the end of 1997, the gas supply to CJC 2 (CJC 1) was done through $6(4)$ inlets at the outer circumference of the chamber which were connected to a single gas supply ring. In the same way the gas was taken out on the other side of the chamber. This setup did not allow the gas flow through the individual gas inlets/outlets to be controlled, nor did it enable separate gas analyses of gas coming from different regions of the chambers. However, the routinely performed gas analyses done with the gas chromatograph, which is part of the gas system, did not show any impurities above the sensitivity of this device of $300 \mathrm{ppm}$. In order to investigate the possibility of an contamination induced from parts of the gas system, a dedicated analysis of gas samples taken during the 1997/1998 shutdown without the chamber in the gas circuit was done using a highly sensitive setup available at CERN [6] involving a mass spectrometer (MSD, sensitivity $\mathcal{O}(\mathrm{ppm})$ ) and an electron capture device $(\mathrm{ECD}$, sensitivity $\mathcal{O}(\mathrm{ppb}))$. This analysis did not yield conclusive results but as a preventive measure the gas supply rings were replaced with individual gas pipes equipped with flow meters in order to guarantee a more homogenous gas distribution within the entire chamber volume. A disadvantage of this modification was a further reduction of the overall gas flow through the chamber (for possible consequences see section 5).

\section{Malter Effect}

Another problem occurred in 1998/1999 when HERA was operated with electrons instead of the previously used positrons leading to significantly increased background levels in the $\mathrm{H} 1$ interaction region. The experience from 1992 to $1997 \mathrm{had}$ shown that under normal conditions the chamber current scaled rather well with the positron current in the HERA-e ring. Therefore, the ratio of

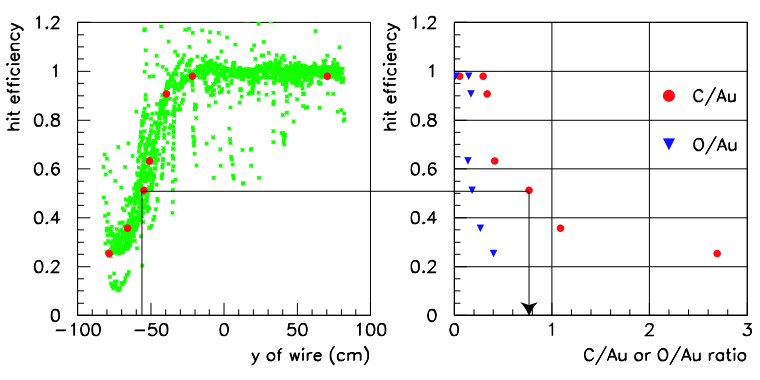

Figure 6. Correlation of hit efficiency with ycoordinate of wires in CJC 2 (a) and with the observed fraction of carbon or oxygen to gold in the corresponding x-ray spectra (b). In (a), wires which have been investigated with the electron microscope are marked with full circles.

the chamber current and the positron (electron) current in HERA is used as a measure of the background conditions. As can be seen in figure 7, where the ratio is plotted as a function of time, the background situation was much worse when HERA was filled with electrons. As a result, in several regions of CJC 1 and CJC 2 sudden steps in the cell current of the order of $1-2 \mu \mathrm{A}$ were observed in 1998. Simultaneously increased currents were also seen in the two adjacent cells on both sides, which immediately disappeared once the central cell was disconnected from high voltage. This was done to prevent more damage for further data taking. Analysis of some data taken with the increased cell current revealed that in a localized area in $r \times \varphi \times z$ no proper chamber signals were recorded. The observations made are consistent with the Malter effect occuring on the cathode wires. As a practical remedy, before an access to the chamber was possible, the $0.1 \%$ additive of $\mathrm{H}_{2} \mathrm{O}$ was replaced with $0.8 \%$ of $\mathrm{C}_{2} \mathrm{H}_{5} \mathrm{OH}$ starting in May 1999 (no attempt was made to increase the water content beforehand). Except for the region where the Malter effect showed up first and therefore an increased current was drawn for an extended period before being noticed, all other regions could be put back into operation again this way. Subsequent visual inspection revealed black deposits on an area of a few $\mathrm{cm}^{2}$ of the cathode wire planes of the problematic areas. 


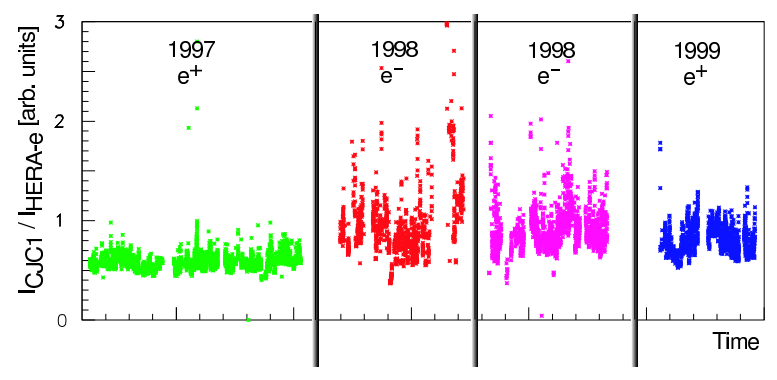

Figure 7. CJC 1 chamber current normalised to the positron- (electron-) current in the HERA-e ring as a function of time showing a strong increase in background when HERA operation was changed from $e^{+} p$ to $e^{-} p$ mode.

\section{Present Status}

At the end of the 2000 data taking period an indication of a possible gain drop was seen again at the bottom of CJC 2. As a preventive measure the sense wires in the lower part of the chamber as well as all wires in a region around the areas where Malter effect had been observed were replaced. In the future CJC 1 and CJC 2 will be connected to identical but separated gas circuits in order to allow an increased gas flow through both chambers.

\section{Summary}

Various kinds of aging effects have been observed in the central jet chamber of the $\mathrm{H} 1$ experiment after moderate integrated charges on the sense wires:

- wire corrosion in CJC 1,

- Malter effect on cathodes of CJC 1 and CJC 2,

- sense wire deposits in lower part of CJC 2.

By modifying sense wire crimp parts, rewiring and changing gas additives the symptoms could be cured. However, a number of questions are still open. Up to now there is no satisfactory explanation why the corrosion process was observed only in CJC 1 or why the sense wire aging only occurred in the lower part of CJC 2. The observations do, however, underline the importance of having control over as many system parameters as possible from the construction of a chamber to its operation.

\section{REFERENCES}

1. H1 collaboration, The H1 Detector at HERA, Nucl. Instr. Meth. A 386 (1997) 310; The Tracking, Calorimeter and Muon Detectors of the H1 Experiment, Nucl. Instr. Meth. A 386 (1997) 348.

2. J. Bürger et al., The Central Jet Chamber of the H1 Experiment, Nucl. Instr. Meth. A 279 (1989) 217.

3. S. Masson, Entwicklung, Bau und Betrieb universeller Anlagen zur Gasversorgung für das innere Spurkammersystem des H1Detektors, Ph.D. Thesis, PITHA94/50, RWTH Aachen (1994).

4. Courtesy of V.O.M. Verbundzentrum für Oberflächen- und Mikrobereichsanalyse Münster, Germany.

5. Courtesy of O. Greis, Zentralbereich Elektronenmikroskopie TUHH, Hamburg-Harburg, Germany.

6. Courtesy of Mar Capeàns, CERN, Geneva, Switzerland. 